\title{
Simple and robust free-electron laser doubler
}

\author{
S. Di Mitri®, ${ }^{1, *}$ G. De Ninno, ${ }^{1,2}$ R. Fabris, ${ }^{1}$ S. Spampinati, ${ }^{1}$ and N. R. Thompson ${ }^{3,4}$ \\ ${ }^{1}$ Elettra-Sincrotrone Trieste S.C.p.A., S.S. 14 km 163,5 in Area Science Park, \\ I-34149 Basovizza, Trieste, Italy \\ ${ }^{2}$ Laboratory of Quantum Optics, University of Nova Gorica, Nova Gorica 5001, Slovenia \\ ${ }^{3}$ ASTeC, STFC Daresbury Laboratory, Daresbury, Warrington, WA44AD Cheshire, United Kingdom \\ ${ }^{4}$ Cockcroft Institute, Sci-Tech Daresbury, Keckwick Lane, Daresbury, \\ Warrington WA44AD, United Kingdom
}

(Received 16 May 2019; published 10 October 2019)

\begin{abstract}
We present the design of a free-electron laser (FEL) doubler suitable for the simultaneous operation of two FEL lines, in either self-amplified spontaneous emission or externally seeded configuration. The doubler relies on the physical selection of two longitudinal portions (beamlets) of a single electron bunch at a low energy and on their spatial separation at high energy. Since the two beamlets are naturally synchronized, FEL pump-FEL probe experiments are enabled when the two photon pulses are sent to the same experimental station. The proposed solution offers improved flexibility of operation compared with existing or designed two-pulse, two-color FEL schemes, as it allows independent control and continuous tunability of the color, timing, intensity, and angle of incidence of the radiation pulses at the user end station. Detailed numerical simulations and experimental results demonstrate its feasibility at the Free Electron laser Radiation for Multidisciplinary Investigations (FERMI) FEL facility.
\end{abstract}

DOI: 10.1103/PhysRevAccelBeams.22.100701

\section{INTRODUCTION}

The past decade has seen the advent of extreme vacuumultraviolet and x-ray free-electron lasers (FELs) for scientific applications [1-6]. Their portfolio includes unprecedented spectral brightness [7], femtosecond pulse duration [8], full transverse and longitudinal coherence [4,5], and two-pulse [9-12], two-color operation [13-16]. Tens of femtosecond pulse duration, longitudinal coherence, and synchronized multicolor operation are essential, for example, in four-wave mixing experiments [17].

Since FELs are driven by a single high-brightness radiofrequency linear accelerator (rf linac) [18], at most two photon beam lines are simultaneously supplied by means of fast magnetic electron beam switches [19,20]. In principle, rf deflecting cavities might further enlarge the FEL fan-out [21]. The time separation of consecutive electron bunches sent to distinct undulator lines spans from $100 \mathrm{~ms}$ to $1 \mu \mathrm{s}$, depending on the linac technology $[22,23]$.

FEL pulses at a shorter time separation ("two-pulse" mode) are all driven by electron bunches passing through the same undulator line. For example, nanosecond delay is

\footnotetext{
*simone.dimitri@elettra.eu
}

Published by the American Physical Society under the terms of the Creative Commons Attribution 4.0 International license. Further distribution of this work must maintain attribution to the author(s) and the published article's title, journal citation, and DOI. obtained with the acceleration of twin electron bunches in consecutive if buckets [24-26]. Twin bunches can be made closer at hundreds of femtosecond separation, but their longitudinal dynamics are tightly coupled $[9,10]$. In seeded FELs, two pulses are generated by two external laser pulses impinging on the same electron bunch at the undulator entrance and typically separated by $100-500 \mathrm{fs}[11,12]$. A series of two-pulse schemes which rely on the manipulation of a single electron bunch through magnetic delay lines (chicanes) [13,14], single intense chirped seed laser pulses [15], and trajectory control in the undulator ("fresh slice") [16] can provide time separation of the FEL pulses up to approximately a picosecond. More recently, the adoption of a double-slotted thin foil for spoiling the beam transverse emittances [27] has been revisited to operate a two-color, two-pulse FEL with some flexibility in temporal (either in the range 100-300 fs or at the picosecond scale) and wavelength separation (from $1 \%$ to $3 \%$ in the $\mathrm{x}$ rays) [13,28-31]. Similar spectrotemporal control was proposed in the presence of additional hardware such as highimpedance corrugated structures [32,33] or a magnetic chicane traversed by an angularly tilted beam [34]. Selfseeded FEL configurations can produce two-color pulses but with very limited, if any, delay control $[35,36]$. State-ofthe-art twin bunch operation allows pump-probe experiments with a few-femtosecond timing jitter [37]. Schemes exploiting a single electron bunch naturally produce two self-synchronized FEL pulses; i.e., the relative timing jitter approaches the subfemtosecond range. 
Each of the aforementioned options has, of course, its own limitations. Schemes based on twin bunches and emittance spoiling show limited tunability of the FEL wavelength, where the spectral separation of the two colors and the individual color tunability is limited by the resonant range of the undulator field. Schemes in which the undulator line is shared by the two pulses ("split undulator") can be limited in intensity, because each FEL pulse is amplified in only half of the line, and a much longer undulator is needed for both pulses to reach saturation $[38,39]$. Fresh slice and split undulators have limited control of the incident angle on the sample, because the source points of the FEL pulses are typically separated by tens of meters, which may compromise the ability of a single photon transport line to optimally transport both pulses to the user end station. This limitation is expected to be alleviated in Ref. [31] but still with reduced flexibility in other FEL parameters. Finally, all the schemes suffer from some limited variability of the angle between the two pulses, which can be very important for applications such as four-wave mixing.

We propose a scheme in which two longitudinal portions of the electron bunch (beamlets) are physically selected with a thick mask at a low energy in the linac (beam scraping) and spatially separated with a septum magnet at a high energy. Each beamlet is then sent to a distinct undulator line. Unlike any of the preceding schemes, ours allows the simultaneous operation of two FEL lines, naturally synchronized at the (sub)femtosecond level, with a continuously tunable relative delay from a few femtoseconds to approximately a picosecond. Since two undulator lines are used, full and independent control of the color, timing, intensity, and angle of incidence of the individual radiation pulses on the sample is ensured. If the two FEL pulses are directed to the same user end station, FEL-pump FEL-probe experiments can be done with unprecedented flexibility, either in self-amplified spontaneous emission $[40,41]$ or in seeded configurations $[42,43]$.

\section{ELECTRON BEAM SCRAPING AND TRANSPORT}

The scheme is sketched in Fig. 1, and typical parameters at the Free Electron laser Radiation for Multidisciplinary Investigations (FERMI) [4,5] are considered in the following as a case study. A high-brightness electron bunch is generated in a photoinjector (gun) and time compressed in a magnetic chicane (BC1). Compression requires that longitudinal portions of the bunch are set to slightly different energies by a proper rf phasing of the upstream linac, e.g., $\delta \approx 4 \%$ peak-to-peak relative energy deviation at the $\mathrm{BC} 1$ mean energy of $270 \mathrm{MeV}$. Their arrival time at the exit of $\mathrm{BC} 1$ is compressed in proportion to $\delta$ by the momentum compaction of the chicane, e.g., $R_{56}=-41 \mathrm{~mm}$ for a maximum energy dispersion function of $\eta_{x}=0.26 \mathrm{~m}$ in

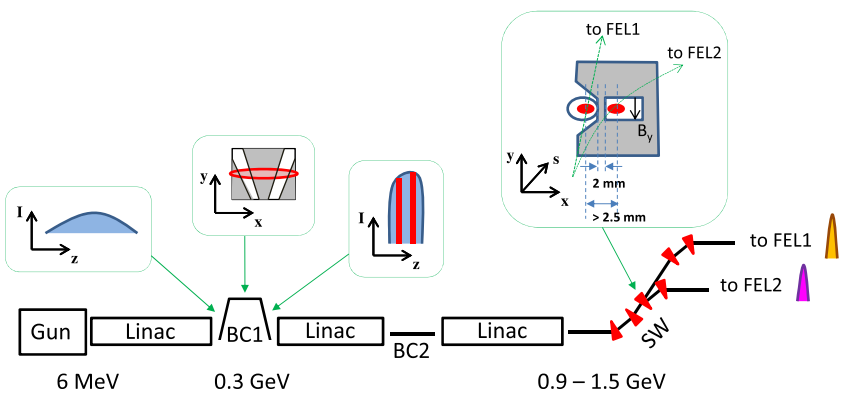

FIG. 1. FEL doubler applied to FERMI (not to scale): Selection of electron beamlets in $\mathrm{BC} 1$ with a mask (red slices; blue regions are not transported downstream) and separation in the switchyard (SW) with a septum magnet.

the middle of the chicane. The bunch length compression factor is

$$
C \equiv \frac{\sigma_{t, i}}{\sigma_{t, f}} \cong\left(1-\frac{R_{56} \sigma_{\delta}}{c \sigma_{t, i}}\right)^{-1}
$$

with $\sigma_{\delta}=2 \%$ the relative energy spread linearly correlated to the initial bunch duration $\sigma_{t, i}=2.8 \mathrm{ps}$.

A mask with two apertures is installed in the middle of $\mathrm{BC} 1$, where the particle horizontal position with respect to the reference trajectory is $x(s) \cong \eta_{x}(s) \delta$, and betatron oscillations can be neglected $\left(\eta_{x} \sigma_{\delta} \cong 5 \mathrm{~mm} \gg \sqrt{\beta_{x} \varepsilon_{x}} \cong\right.$ $0.1 \mathrm{~mm}$, with $\beta_{x}$ the betatron function at the mask location and $\varepsilon_{x}$ the beam geometric emittance). The mask, made of $\sim 10-$ mm-thick copper, acts like a scraper: It physically selects two transversally displaced beamlets, the rest of the bunch being scattered at large angles and absorbed in the chamber. The feasibility of such beam scraping while preserving the brightness of the selected beamlets was demonstrated at FERMI [44] and is routinely adopted at Linac Coherent Light Source (LCLS) for FEL optimization [45]. Since the chicane is achromatic, the two beamlets exit BC1 separated both in energy and in time but spatially aligned. With V-shaped geometry [16], the vertical position of the mask determines both the width of the two apertures and their transverse separation. The beamlet duration at the exit of $\mathrm{BC} 1$, as well as their time separation, is estimated by [33]

$$
\Delta t_{\mathrm{FWHM}} \approx \frac{2 \Delta x}{\eta_{x}} \frac{\sigma_{t, i}}{C \sigma_{\delta}},
$$

with $\Delta x$ either the aperture width or the width of the central slit, respectively. For example, with $C=10$ and $\Delta x=$ $3 \mathrm{~mm}, \Delta t_{\mathrm{FWHM}} \approx 320 \mathrm{fs}$. Although $\mathrm{BC} 1$ only is used in our example, the scheme also works in a multistage compression system. It is worth noting that the mask acts as an energy bandpass filter; i.e., it tends to remove any beam energy jitter accumulated up to $\mathrm{BC} 1$. 


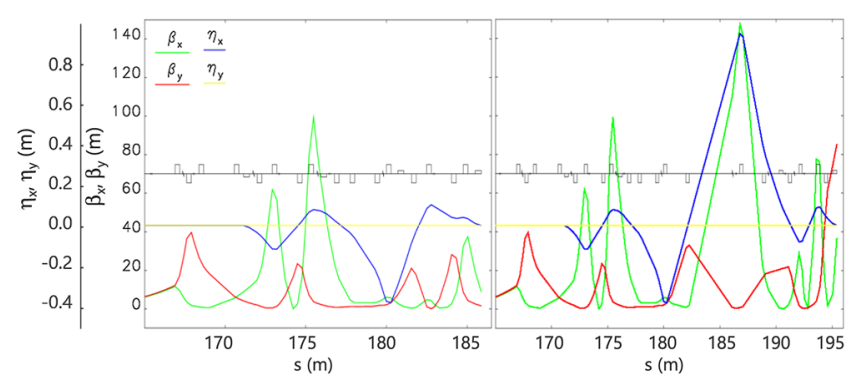

FIG. 2. Betatron and dispersion functions from the FERMI linac end to the switchyard end, for the FEL2 (left) and FEL1 beam lines. The septum is placed in proximity of the maximum negative dispersion peak $(s=180 \mathrm{~m})$. Quadrupole magnets are in gray.

Downstream of $\mathrm{BC} 1$, the linac rf phases are adjusted to ensure both a large relative energy offset of the beamlets $\left(\delta_{f}\right)$, which is suitable for their spatial separation in the switchyard, and a small energy spread in each beamlet $\left(\sigma_{\delta, f}\right)$, as required for efficient lasing. The $\mathrm{rf}$ phasing takes into account the effect of the longitudinal wakefields excited by the leading beamlet on the trailing one $[46,47]$. For example, we obtain in simulation $\delta_{f}=$ $0.9 \%$ and $\sigma_{\delta, f}=0.04 \%$. Doing so, the final mean energy is lowered from $1.40 \mathrm{GeV}$ for the standard whole bunch preparation to $1.25 \mathrm{GeV}$ (see later Fig. 3).

The FERMI switchyard (SW in Fig. 1) is a 40-m-long line working in the energy range $0.9-1.5 \mathrm{GeV}$. It comprises two branches, each including two modified double-bend achromatic cells. The first cell is in common, and the dipole bending angle is $3^{\circ}$. The optics is set for preservation of the beam emittance in the presence of coherent synchrotron radiation [48]. The two branches lead to the FEL1 and FEL2 undulator lines; these are parallel and separated by $1 \mathrm{~m}$. Depending on the electron beam energy and on the resonant harmonic jump set by the variable gap undulators, FERMI covers the fundamental wavelength range $20-100 \mathrm{~nm}$ with FEL1 [4] and 4-20 nm with FEL2 [5], in the high-gain harmonic generation (HGHG) mode of operation [42].

For the purpose of separating the beamlets in the bending plane, the SW optics was modified. A dispersion function as large as $\eta_{x}=-0.3 \mathrm{~m}$ is generated at the location of the third dipole magnet, i.e., at the entrance to the FEL2 branch line (see Fig. 2). The dipole magnet would be replaced by a thin septum magnet, having a similar length of $0.5 \mathrm{~m}$ and the same bending angle. The beamlet at a low-energypositive- $x$ coordinate is bent by the septum magnetic field and directed towards FEL2. The other beamlet samples a residual $\sim 100 \mathrm{Gm}$ integrated field, which is easily compensated by steering magnets. This beamlet continues its straight path towards the next double-bend cell and is eventually directed to FEL1. Quadrupole magnets are installed in a nondispersive region in front of the undulator for optics matching to the undulator. In order for the two beamlets to safely reach the present common dump at the

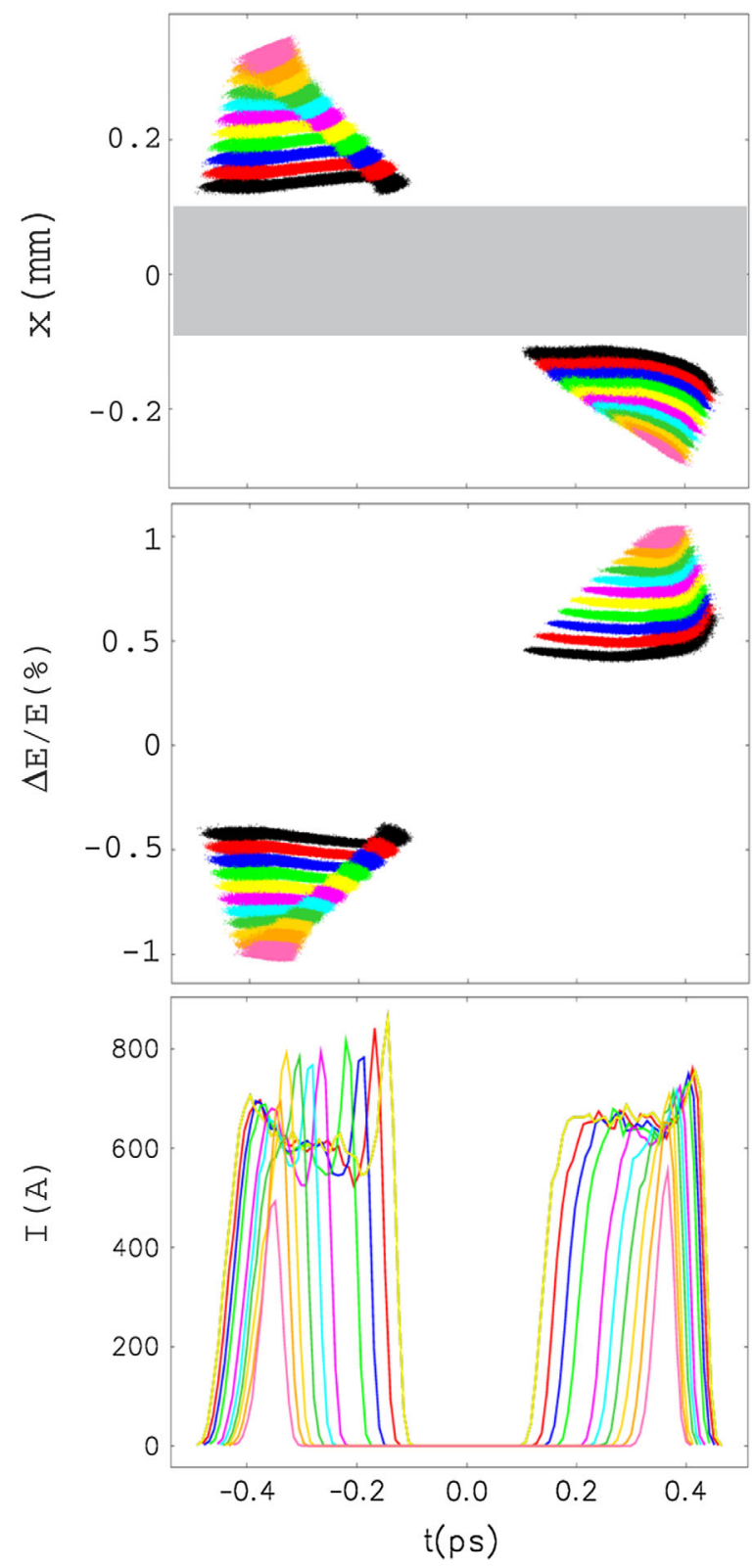

FIG. 3. From top to bottom, longitudinal phase space, top view (with the shadow of the $2 \mathrm{~mm}$ septum thickness) and current profile of the two beamlets at the undulator entrance, for a mask slit width in the range 3-8 $\mathrm{mm}$ and aperture width of $1-6 \mathrm{~mm}$. The bunch head is on the left. The linac rf phases were optimized for one beamlet's duration only (black).

end of the undulators [49], the FERMI dump line would be modified. This modification is not required in facilities where multiple dumps downstream of distinct undulators are already available.

Figure 3 shows the beamlet longitudinal phase spaces at the entrance of the septum magnet, for different separations of the apertures in the mask. Particle tracking was carried out with the elegant code [50], including all major collective effects [47,51] from the injector exit to the undulator. The main beam and mask parameters are listed 
TABLE I. Electron beam parameters at the entrance of $\mathrm{BC} 1$ (whole bunch) and of the undulator (each beamlet) as from the tracking run. The mask geometry is also reported.

\begin{tabular}{lccc}
\hline \hline Quantity & @ BC1 & @ UND & Units \\
\hline Charge & 0.7 & $\sim 0.2$ & $\mathrm{nC}$ \\
Mean energy & 0.27 & 1.25 & $\mathrm{GeV}$ \\
Relative energy spread, rms & 2.0 & $<0.03$ & $\%$ \\
Duration, FWHM & 10.8 & 0.3 & $\mathrm{ps}$ \\
Peak current (core) & 650 & 650 & $\mathrm{~A}$ \\
Horizontal normalized emittance, & 0.6 & 0.7 & $\mu \mathrm{m}$ \\
$\quad$ projected rms & & & \\
$\quad$ Vertical normalized emittance, & 0.6 & 0.6 & $\mu \mathrm{m}$ \\
$\quad$ projected rms & & & \\
Mask slit width & 1 & & $\mathrm{~mm}$ \\
Mask aperture width & 3 & & $\mathrm{~mm}$ \\
\hline \hline
\end{tabular}

in Table I. In this simulation, the outer borders of the mask apertures are kept fixed, so that a larger aperture separation (larger energy offset of the two beamlets) implies a smaller aperture width (shorter beamlet duration). Figure 3 also shows the corresponding horizontal separation of the beamlets at the septum entrance and their current profile. Alternatively, since the FWHM horizontal beam size at the mask is $20 \mathrm{~mm}$, a fixed aperture width of, e.g., $0.1,1$. or $5 \mathrm{~mm}$ could produce 11-, 108-, or 538-fs-long beamlets, respectively, at the maximum separation of $\sim 2$ ps at the undulator.

The horizontal separation of the beamlets at the septum entrance is $\eta_{x} \delta_{f} \geq 2.5 \mathrm{~mm}$ and much larger than their individual betatron beam size, $\sqrt{\beta_{x} \varepsilon_{x}} \cong 0.1 \mathrm{~mm}$. We thus consider a minimum septum thickness of $2 \mathrm{~mm}$, which can be provided by an in-vacuum eddy-current septum magnet [52-54]. We developed a septum design of $15 \times 25 \mathrm{~mm}^{2}$ transversal acceptance. A maximum electric power of $\sim 100 \mathrm{~W}$ is expected to be safely dissipated, which translates into a repetition rate of $25 \mathrm{~Hz}$ at the beamlets' mean energy of $1.25 \mathrm{GeV}$. The beamlets' rms position jitter at the septum must be much smaller, say one-tenth, of $2 \mathrm{~mm}$, which implies a relative rms energy jitter of $0.07 \%$ and an overall trajectory jitter $\leq 50 \mu \mathrm{m}$. This error budget is well within reach of x-ray FEL facilities [55].

The optics of the switchyard branches is achromatic. Although it is not isochronous $\left(R_{56}=-0.3 \mathrm{~mm}\right.$ for FEL1 and $+2.9 \mathrm{~mm}$ for FEL2), the beamlets' duration is almost unchanged by virtue of their negligible correlated energy spread, i.e., $\Delta \sigma_{t} \cong R_{56} \sigma_{\delta, f} / c \leq 4$ fs. The minimum relative delay of the beamlets at the undulator is determined by the difference in the transfer matrix of the two branches: $\Delta t \cong$ $\left(R_{51} \Delta x+R_{52} \Delta x^{\prime}+R_{56} \delta\right) / c \cong\left(R_{51} \eta_{x} \delta+R_{52} \eta_{x}^{\prime} \delta+R_{56} \delta\right) /$ $c=(67+5+75) \mathrm{fs}=147 \mathrm{fs}$ in our case.

In general, depending on the geometry of the switchyard and with alternative optics designs, the beamlets' time separation can be tuned from $\sim 10$ fs to approximately a picosecond. On top of this, split-and-delay manipulation of the photon beams could enable time separation up to tens of picoseconds, though at the expense of flux reduction $[56,57]$. The relative rms timing jitter of the two beamlets at the undulators is estimated as $\delta_{t} \approx\left|R_{56}\right| \delta_{r} /(2 c)$, with $\delta_{r}$ their rms relative energy jitter. Since the two beamlets are originated by the same bunch, $\delta_{r}$ is nothing else but the energy variation of the bunch head with respect to the bunch tail, evaluated pulse to pulse, for the compressed bunch. By virtue of the intrapulse rf stability of the FERMI linac [58,59], $\delta_{r}$ is dominated by the jitter of the bunch duration (i.e., the variation of the temporal separation of the bunch head and tail, which will be sampling slightly different rf phases on consecutive shots). This is usually at the few percent level, i.e., a few tens of femtoseconds [60]. For typical near-to-crest acceleration after compression, that translates into $\delta_{r} \leq 0.01 \%$. Considering $\left|R_{56}\right| \approx 3 \mathrm{~mm}$, we get $\delta_{t} \leq 0.5$ fs. $\delta_{t}$ could be made even smaller by an isochronous optics design of the switchyard lines.

\section{LASING}

Figure 4 shows the result of time-dependent FEL1 (HGHG) and FEL2 simulations (cascaded HGHG) done with the Genesis 1.3 code [61], for the mask geometry and beam parameters in Table I; the phase space of the beamlets from particle tracking is shown in Fig. 3 (black). The mask was chosen so as to make the beamlets long enough, approximately 300 fs full width, to accommodate an external seeding laser of $50 \mathrm{fs}$. The FEL input and output parameters are summarized in Table II. The HGHG FEL output shows that the brightness of the beamlets is high enough to reach saturation in six radiator modules, thus to lase efficiently in the typical FERMI wavelength range.

In order to demonstrate the preservation of the beamlet brightness for lasing, we conducted an experiment with beam and mask parameters close to those in Table I but a single mask aperture as due to available hardware. Figure 5 shows the measured spectrum of the first HGHG stage of FERMI FEL2, tuned at the eighth harmonic of the seed laser wavelength. The seed laser duration was about $50 \mathrm{fs}$. The spectrum is measured as a function of the delay of the seed laser relative to the electron bunch arrival time. The top plot is without beam scraping; the bottom plot is for
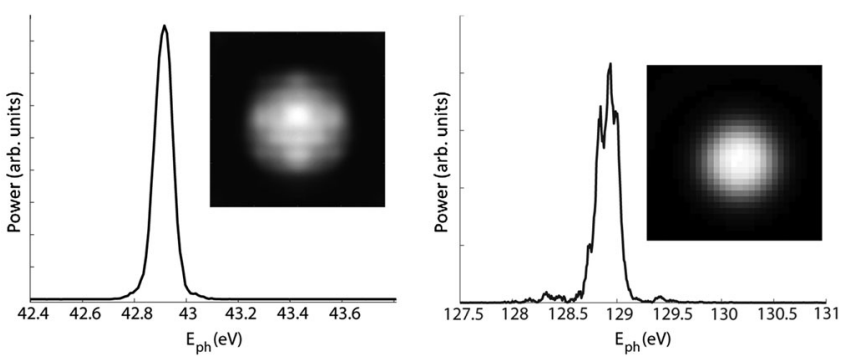

FIG. 4. Spectral power and transverse intensity distribution (inset) at the end of the FEL1 (left) and FEL2 undulator line. Genesis 1.3 simulation. Electron beamlet parameters as in Table I. 
TABLE II. FEL1 and FEL2 input and output parameters. Electron beamlet parameters as in Table I.

\begin{tabular}{lccc}
\hline \hline Quantity & FEL1 & FEL2 & Units \\
\hline Seed laser pulse energy & 5 & 10 & $\mu \mathrm{J}$ \\
Seed laser duration, FWHM & 50 & 50 & $\mathrm{fs}$ \\
Harmonic jump & 9 & 27 & \\
Central wavelength & 28.8 & 9.6 & $\mathrm{~nm}$ \\
Relative bandwidth, FWHM & 0.20 & 0.18 & $\%$ \\
Pulse energy & 60 & 13 & $\mu \mathrm{J}$ \\
Pulse duration, FWHM & 30 & 37 & $\mathrm{fs}$ \\
Peak power & 2.0 & 0.4 & $\mathrm{GW}$ \\
\hline \hline
\end{tabular}

scraping in $\mathrm{BC} 1$ set to generate a beamlet duration of approximately $330 \mathrm{fs}$. The extension of the lasing region as a function of the seed laser-electron bunch delay confirms the expected beamlet duration, and it highlights a region of efficient lasing in the beamlet as long as $\sim 150 \mathrm{fs}$.
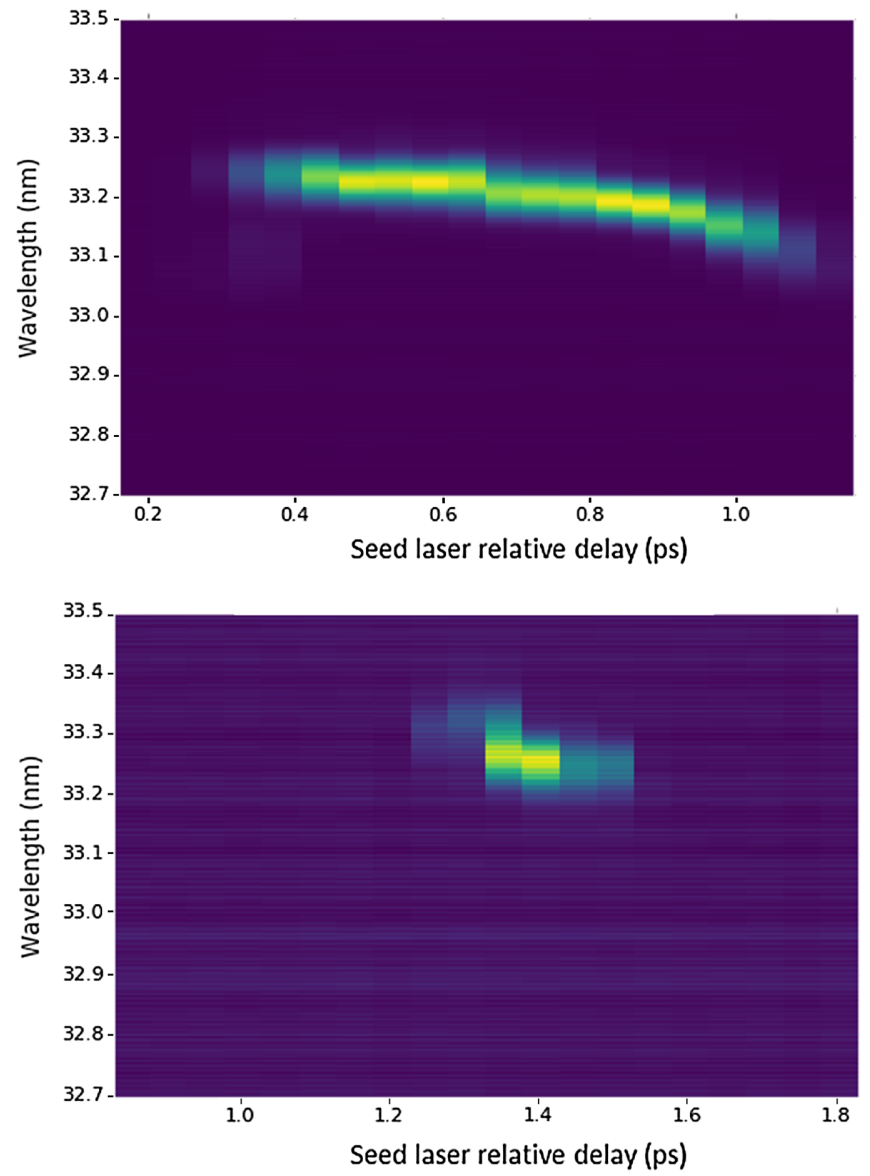

FIG. 5. Spectrum of the first stage of FERMI HGHG FEL2 vs seed laser delay. The seed laser is superimposed to the whole electron beam (top) and to a single beamlet produced with scraping in BC1 (bottom). The spectrum intensity is normalized in both plots to the peak value. The seed is time shifted in steps of $50 \mathrm{fs} ; 20$ shots are consecutively recorded for each delay value [62].
The spectrum intensity is normalized to the peak value in both plots: The average FEL pulse energy was $35 \mu \mathrm{J}$ for the whole beam and $15 \mu \mathrm{J}$ for the selected beamlet without further optimization of the spatial and temporal overlap of the seed laser and electron beamlet.

\section{FINAL REMARKS}

In conclusion, we have demonstrated with detailed numerical simulations that two-pulse, two-color FEL emission synchronized at the subfemtosecond level can be generated by splitting the electron bunch in two beamlets and that these can be safely sent to distinct undulator lines. The scheme is suitable for the simultaneous operation of experimental beam lines receiving FEL pulses generated by very similar electron beam parameters and can be implemented at existing facilities with limited cost and reduced impact on the infrastructure.

Unlike any HGHG option, the proposed scheme has no color limitation due to the harmonic up-conversion of the seed laser wavelength. Accordingly, this study is expected not only to pave the way to simultaneous operation of two synchronized FEL lines, but also to more flexible, robust, and reliable two-color, two pulse schemes for, e.g., fourwave mixing spectroscopy as well as a broader variety of FEL-pump FEL-probe experiments, including transient grating spectroscopic methods [63]. Since the pump and probe are generated with two different undulators, and for a relative time separation of the two pulses up to $1 \mathrm{ps}$ or so, there is no need for a large split-and-delay system for the photon beam, which can be costly and difficult to operate and reduces the photon flux at the sample.

At FERMI, a relative delay of the two pulses in the range 200-600 fs can be achieved with little modification to the switchyard footprint, at $0.65 \mathrm{kA}$ peak current level. However, an additional small chicane in one of the two beam lines could allow the relative delay of the two FEL pulses to go through zero. A recent experimental proof of beam scraping for the production of a single beamlet with beam parameters as in Table I and standard optics in the switchyard has been collected and lasing demonstrated from the first stage of FEL2. Additional tests are ongoing to further optimize and characterize such promising results. By virtue of electron beam scraping, $\sim 10-$ fs-long beamlets can be provided at the undulator. Thus, in principle, seed laser pulses longer than the beamlet can stimulate FEL emission of few-femtoseconds-long, fully coherent photon pulses from UV to soft $\mathrm{x}$ rays.

For future facilities with freedom of parameter choice, the two beamlets could be created using a double photoinjector laser pulse, accelerated at the same phase on different rf cycles, before being given small energy offsets in a subharmonic cavity so that they can be separated into two FEL beam lines by the septum with the same scheme presented above. Such a double-pulse option may offer some more flexibility in beam compression and avoids 
relatively large beam power losses induced by scraping at high repetition rates.

High repetition rates also limit the performance of the in-vacuum septum magnet: a maximum safe value of the dissipated average electric power $\left\langle P_{d}\right\rangle \sim 100 \mathrm{~W}$ implies, e.g., bending angles $\theta=1^{\circ}-3^{\circ}$, beam energies $E_{b}=$ $1-5 \mathrm{GeV}$, and consequently pulse frequency $f_{\text {rep }}=$ $10-300 \mathrm{~Hz}$, where $\left\langle P_{d}\right\rangle \propto \theta^{2} E_{b}^{2} f_{\text {rep. Reliability at the }}$ highest repetition rates would also require a specific study of the circuit components. Alternatively, $\left\langle P_{d}\right\rangle>100 \mathrm{~W}$ can be dissipated with an out-of-vacuum dc septum magnet; a minimum thickness of $\sim 5 \mathrm{~mm}$ should then be considered. In our case study, such a thickness would force to either $\delta_{f}>2 \%$ or $\left|\eta_{x}\right|>0.7 \mathrm{~m}$. However, a larger energy separation would be obtained at the expense of a lower mean energy of the beamlets (because of more pronounced off-crest acceleration in the linac), while a larger dispersion would need a revision of the FERMI switchyard layout. We conclude that FEL repetition rates $\geq 100 \mathrm{~Hz}$ imply larger beamlet separation; this is affordable in FELs with a larger linac energy budget (allowing larger $\delta_{f}$ ) and/or a longer switchyard (allowing smaller $\theta$ ) than considered in our case study.

\section{ACKNOWLEDGMENTS}

One of the authors (S. D. M.) acknowledges Dr. M. Aiba for helpful information on septum magnets, M. Milloch for a discussion about energy jitter in the FERMI linac, and Dr. W. Fawley for useful comments about the FEL doubler. The FERMI team is acknowledged for support to the FEL operation. This work has received funding by the European Union's Horizon 2020 research and innovation program under Grant Agreement No. 777431.

[1] W. Ackermann et al., Operation of a free-electron laser form the extreme ultraviolet to the water window, Nat. Photonics 1, 336 (2007).

[2] P. Emma et al., First lasing and operation of an angstrom wavelength free-electron laser, Nat. Photonics 4, 641 (2010).

[3] T. Ishikawa et al., A compact x-ray free-electron laser emitting in the sub-angstrom region, Nat. Photonics 6, 540 (2012).

[4] E. Allaria et al., Highly coherent and stable pulses from the FERMI seeded free-electron laser in the extreme ultraviolet, Nat. Photonics 6, 699 (2012).

[5] E. Allaria et al., Two-stage seeded soft-x-ray free-electron laser, Nat. Photonics 7, 913 (2013).

[6] H.-S. Kang et al., Hard x-ray free-electron laser with femtosecond-scale timing jitter, Nat. Photonics 11, 708 (2017).

[7] E. A. Seddon et al., Short-wavelength free-electron laser sources and science: A review, Rep. Prog. Phys. 80, 115901 (2017).
[8] A. A. Lutman, M. W. Guetg, T. J. Maxwell, J. P. MacArthur, Y. Ding, C. Emma, J. Krzywinski, A. Marinelli, and Z. Huang, High-Power Femtosecond Soft X Rays form FreshSlice Multi-Stage Free-Electron Lasers, Phys. Rev. Lett. 120, 264801 (2018).

[9] A. Marinelli et al., High-intensity double-pulse x-ray freeelectron laser, Nat. Commun. 6, 6369 (2015).

[10] A. Petralia et al., Two-Color Radiation Generated in a Seeded Free-Electron Laser with Two Electron Beams, Phys. Rev. Lett. 115, 014801 (2015).

[11] E. Allaria et al., Two-colour pump-probe experiments with a twin-pulse-seed extreme ultraviolet free-electron laser, Nat. Commun. 4, 2476 (2013).

[12] E. Ferrari et al., Widely tunable two-colour seeded freeelectron laser source for resonant-pump resonant-probe magnetic scattering, Nat. Commun. 7, 10343 (2016).

[13] A. A. Lutman, R. Coffee, Y. Ding, Z. Huang, J. Krzywinski, T. Maxwell, M. Messerschmidt, and H.-D. Nuhn, Experimental Demonstration of Femtosecond Two-Color X-Ray Free-Electron Lasers, Phys. Rev. Lett. 110, 134801 (2013).

[14] T. Hara et al., Two-color hard x-ray free-electron laser with wide tunability, Nat. Commun. 4, 2919 (2013).

[15] G. De Ninno, B. Mahieu, E. Allaria, L. Giannessi, and S. Spampinati, Chirped Seeded Free-Electron Lasers: SelfStanding Light Sources for Two-Color Pump-Probe Experiments, Phys. Rev. Lett. 110, 064801 (2013).

[16] A. A. Lutman et al., Fresh-slice multicolor x-ray freeelectron lasers, Nat. Photonics 10, 745 (2016).

[17] F. Bencivenga et al., Four-wave mixing experiments with extreme ultraviolet transient gratings, Nature (London) 520, 205 (2015).

[18] S. Di Mitri and M. Cornacchia, Electron beam brightness in linac drivers for free-electron-lasers, Phys. Rep. 539, 1 (2014).

[19] B. Faatz et al., Simultaneous operation of two soft x-ray free-electron lasers driven by one linear accelerator, New J. Phys. 18, 062002 (2016).

[20] T. Hara et al., Pulse-by-pulse multi-beam-line operation for x-ray free-electron lasers, Phys. Rev. Accel. Beams 19, 020703 (2016).

[21] M. Placidi, J.-Y. Jung, A. Ratti, and C. Sun, Compact spreader schemes, Nucl. Instrum. Methods Phys. Res., Sect. A 768, 14 (2014).

[22] R. Brinkmann, E. A. Schneidmiller, J. Sekutowicz, and M. V. Yurkov, Prospects for CW operation of the European XFEL in hard X-ray regime, in Proceedings of the 36th International Free Electron Laser Conference, Basel, Switzerland, 2014 (JACoW, 2014), MOP067.

[23] J. N. Galayda, The LCLS-II: A high power upgrade to the LCLS, in Proceedings of the 9th International Particle Accelerator Conference, Vancouver, Canada, 2018 (2018).

[24] F.-J. Decker et al., A demonstration of multi-bunch operation in the LCLS, in Proceedings of the 32nd Free Electron Laser Conference, Malmö, Sweden (Max-lab, Sweden, 2010), WEPB33.

[25] M. H. Seaberg et al., Nanosecond X-Ray Photon Correlation Spectroscopy on Magnetic Skyrmions, Phys. Rev. Lett. 119, 067403 (2017). 
[26] G. Penco et al., Two-bunch operation with ns temporal separation at the FERMI FEL facility, New J. Phys. 20, 053047 (2018).

[27] P. Emma, K. Bane, M. Cornacchia, Z. Huang, H. Schlarb, G. Stupakov, and D. Walz, Femtosecond and Subfemtosecond X-Ray Pulses from a Self-Amplified SpontaneousEmission-Based Free-Electron Laser, Phys. Rev. Lett. 92, 074801 (2004).

[28] Z. Zhang, Y. Ding, P. Emma, Z. huang, A. Marinelli, and C. Tang, Flexible pulse delay control up to picosecond for high-intensity twin electron bunches, Phys. Rev. Accel. Beams 18, 090701 (2015).

[29] C. Feng, Z. Wang, X. Wang, and D. Huang, Generation of two-color ultra-short radiation pulses from two electron bunches and a chirped seeded free-electron laser, Nucl. Instrum. Methods Phys. Res., Sect. A 807, 79 (2016).

[30] Y. Ding et al., Generating femtosecond x-ray pulses using an emittance-spoiling foil in free-electron lasers, Appl. Phys. Lett. 107, 191104 (2015).

[31] Á.S. Hernández, E. Prat, and S. Reiche, Generation of two-color X-ray free-electron-laser pulses from a beam with a large energy chirp, and a slotted foil, Phys. Rev. Accel. Beams 22, 030702 (2019).

[32] S. Bettoni, E. Prat, and S. Reiche, Two-color beam generation based on wakefield excitation, Phys. Rev. Accel. Beams 19, 050702 (2016).

[33] W. Qin, Y. Ding, A. A. Lutman, and Y.-C. Chao, Matching based fresh-slice method for generating two-color X-ray free-electron lasers, Phys. Rev. Accel. Beams 20, 090701 (2017).

[34] S. Reiche and E. Prat, Two-color operation of a free electron laser with a tilted beam, J. Synchrotron Radiat. 23, 869 (2016).

[35] G. Geloni, V. Kocharyan, and E. Saldin, Generation of doublet spectral lines at self-seeded x-ray FELs, Opt. Commun. 284, 3348 (2011).

[36] A. A. Lutman, F.-J Decker, J. Arthur, M. Chollet, Y. Feng, J. Hastings, Z. Huang, H. Lemke, H.-D. Nuhn, A. Marinelli, J. L. Turner, S. Wakatsuki, J. Welch, and D. Zhu, Demonstration of Single-Crystal Self-Seeded TwoColor X-Ray Free-Electron Lasers, Phys. Rev. Lett. 113, 254801 (2014).

[37] K. R. Ferguson et al., Transient lattice contraction in the solid-to-plasma transition, Sci. Adv. 2, e1500837 (2016).

[38] G. Geloni, V. Kocharyan, and E. Saldin, DESY Reports No. 10-004 and No. 10-006, 2010.

[39] A. Marinelli, A. A. Lutman, J. Wu, Y. Ding, J. Krzywinski, H.-D. Nuhn, Y. Feng, R. N. Coffee, and C. Pellegrini, Multicolor Operation and Spectral Control in a GainModulated X-Ray Free-Electron Laser, Phys. Rev. Lett. 111, 134801 (2013).

[40] A. M. Kondratenko and E. L. Saldin, Generation of coherent radiation by a relativistic electron beam in an ondulator, Part. Accel. 10, 207 (1980).

[41] R. Bonifacio, C. Pellegrini, and L. M. Narducci, Collective instabilities and high-gain regime in a free electron laser, Opt. Commun. 50, 373 (1984).

[42] L.-H. Yu, Generation of intense uv radiation by subharmonically seeded single-pass free-electron lasers, Phys. Rev. A 44, 5178 (1991).
[43] G. Stupakov, Using the Beam-Echo Effect for Generation of Short-Wavelength Radiation, Phys. Rev. Lett. 102, 074801 (2009).

[44] S. Di Mitri, D. Castronovo, I. Cudin, and L. Fröhlich, Electron slicing for the generation of tunable femtosecond soft x-ray pulses from a free electron laser and slice diagnostics, Phys. Rev. Accel. Beams 16, 042801 (2013).

[45] Y. Ding et al., Beam shaping to improve the free-electron laser performance at the Linac Coherent Light Source, Phys. Rev. Accel. Beams 19, 100703 (2016).

[46] Z. Zhang, Y. Ding, A. Marinelli, and Z. Huang, Longitudinal dynamics of twin electron bunches in the Linac Coherent Light Source, Phys. Rev. Accel. Beams 18, 030702 (2015).

[47] S. Di Mitri, C. Venier, R. Vescovo, and L. Sturari, Wakefield benchmarking at a single-pass high brightness electron linac, Phys. Rev. Accel. Beams 22, 014401 (2019).

[48] S. Di Mitri, M. Cornacchia, and S. Spampinati, Cancellation of Coherent Synchrotron Radiation Kicks with Optics Balance, Phys. Rev. Lett. 110, 014801 (2013).

[49] S. Di Mitri et al., Coherent $\mathrm{THz}$ emission enhanced by coherent synchrotron radiation instability, Sci. Rep. 8, 11661 (2018).

[50] M. Borland, Elegant: A flexible SDDS-compliant code for accelerator simulation, Advanced Photon Source Technical Note No. LS-287, 2000.

[51] M. Borland, Simple method for particle tracking with coherent synchrotron radiation, Phys. Rev. Accel. Beams 4, 070701 (2001).

[52] M. J. Barnes, J. Borburgh, B. Goddard, and M. Hourican, Injection and extraction magnets: Septa, CAS Yellow Report No. CERN-2010-004, 2010, pp. 167-184 [arXiv: 1103.1062].

[53] M. Abliz et al., Septum magnet design for the APS-U, in Proceedings of the North American Particle Accelerator Conference 2016, Chicago, IL (2016), THPOA63, ISBN 978-3-95450-180-9.

[54] N. Tsoupas, K. Brown, F. Meot, C. Pearson, P. Pile, V. Ptitsyn, and A. Rusek, A state of the art current-septum dipole magnet, Nucl. Instrum. Methods Phys. Res., Sect. A 923, 8 (2019).

[55] L. Wang et al., Energy jitter minimization at LCLS, in Proceedings of the 37th International Free Electron Laser Conference, Daejeon, Korea (2015), TUP070.

[56] S. Roling and H. Zacharias, Split-and-delay units for soft and hard X-rays, edited by E. Jaeschke, S. Khan, J. Schneider, and J. Hastings, in Synchrotron Light Sources and Free-Electron Lasers (Springer, New York, 2016), ISBN 978-3-319-04507-8.

[57] W. Lu, B. Friedrich, T. Noll, K. Zhou, J. Hallmann, G. Ansaldi, T. Roth, S. Serkez, G. Geloni, A. Madsen, and S. Eisebitt, Development of a hard x-ray split-and-delay line and performance simulations for two-color pump-probe experiments at the European XFEL, Rev. Sci. Instrum. 89, 063121 (2018).

[58] A. Fabris et al., The LLRF system for the S-band rf plants of the FERMI linac, IEEE Trans. Nucl. Sci. 63, 861 (2016).

[59] P. Craievich, S. Di Mitri, M. Milloch, G. Penco, and F. Rossi, Modeling and experimental study to identify 
arrival-time jitter sources in the presence of a magnetic chicane, Phys. Rev. Accel. Beams 16, 090401 (2013).

[60] M. Veronese, R. Appio, P. Craievich, and G. Penco, Absolute Bunch Length Measurement Using Coherent Diffraction Radiation, Phys. Rev. Lett. 110, 074802 (2013).

[61] S. Reiche, Genesis 1.3: A fully 3D time-dependent FEL simulation code, Nucl. Instrum. Methods Phys. Res., Sect. A 429, 243 (1999).
[62] E. Allaria, E. Ferrari, E. Roussel, and L. Vidotto, REALTA and pyDART: A set of programs to perform real time acquisition and on-line analysis at the FERMI free electron laser, in Proceedings of ICALEPCS2017, Barcelona, Spain (2017), THPHA044.

[63] K. A. Nelson, R. J. Miller, D. R. Lutz, and M. D. Fayer, J. Appl. Phys. 53, 1144 (1982). 